\title{
Valores de presión espiratoria mantenida en la vía aérea como indicador de tolerancia al uso de válvula de fonación en pacientes traqueostomizados
}

\author{
GREGORY VILLARROEL S.*, YORSCHUA JALIL C.*, GONZALO MOSCOSO A.*, \\ PATRICIO BARAÑAO G.*, CLAUDIA ASTUDILLO M.**, \\ BERNARDITA CHATEAU I.**,*** y MIREYA MÉNDEZ R.**,***,
}

Maintained expiratory airway pressure values as an indicator of tolerance of speaking valve in tracheostomized patients

Introduction: Speaking valve (SV) is an unidirectional flow device installed over the tracheostomy tube allowing phonation. Tolerance to this device depends on the permeability of the upper airway (UA), which may be indirectly assessed by measuring UA maintained expiratory pressure (PEMant). Objective: To evaluate the usefulness of the maintained expiratory pressure as a clinical indicator of tolerance to the SV. Method: Twenty three tracheostomized patients (median age 22 months-old) were evaluated with an aneroid manometer during 15 minutes, recording PEMant, arterial oxygen saturation $\left(\mathrm{SaO}_{2}\right)$, heart rate, respiratory rate, accessory muscle use and wheezing as signs of respiratory distress Results: PEMant values less than $10 \mathrm{cmH}_{2} \mathrm{O}$ are associated with tolerance of the SV and values over $20 \mathrm{cmH}_{2} \mathrm{O}$ are associated with intolerance. Conclusion: Values under $10 \mathrm{cmH}_{2} \mathrm{O}$ of PEMant can be used as an indicator of tolerance to VF.

Key words: Maintained expiratory pressure, speaking valve, tracheostomy, airway pressure, upper airway permeability, children.

\section{Resumen}

Introducción: La válvula de fonación (VF), es un dispositivo de flujo unidireccional instalado sobre la cánula de traqueostomía posibilitando la fonación. La tolerancia a este dispositivo depende de la permeabilidad de la vía aérea superior (VAS), pudiendo ser valorada indirectamente a través de la medición de la presión espiratoria mantenida (PEMant) en vía aérea. Objetivo: Estudiar esta técnica como indicador clínico de tolerancia a la VF. Método: Se evaluaron 23 pacientes traqueostomizados (mediana de edad 22 meses) con un manómetro aneroide durante 15 minutos, registrando PEMant, saturación arterial de oxígeno $\left(\mathrm{SaO}_{2}\right)$, frecuencia cardiaca, frecuencia respiratoria, uso de musculatura accesoria y sibilancias para valorar la dificultad respiratoria. Resultados: Valores de PEMant menores a $10 \mathrm{cmH}_{2} \mathrm{O}$ se asocian con tolerancia a la VF y valores sobre $20 \mathrm{CmH}_{2} \mathrm{O}$ a intolerancia a ésta. Conclusión: Valores bajo $10 \mathrm{CmH}_{2} \mathrm{O}$ de PEMant pueden ser indicadores de tolerancia al uso de VF.

Palabras clave: Presión espiratoria mantenida, válvula de fonación, presión de vía aérea, permeabilidad de vía aérea superior, niños.

\footnotetext{
* Kinesiólogo, Hospital Josefina Martínez. Puente Alto. Santiago, Chile.

** Médico Pediatra Broncopulmonar, Hospital Josefina Martínez.

*** Instructor asociado, Facultad de Medicina, Pontificia Universidad Católica de Chile.
} 


\section{Introducción}

Los avances en el tratamiento de enfermedades críticas han determinado que un mayor número de pacientes requiera la utilización de una vía aérea artificial y soporte ventilatorio prolongado ${ }^{1}$.

La decisión de realizar una traqueostomía (TQT) debe ser individualizada, evaluando las necesidades del paciente, el curso de recuperación esperada, el riesgo de intubación continua y riesgos quirúrgicos asociados. Este procedimiento se indica en obstrucción crítica de la vía aérea superior o cuando existe necesidad de ventilación mecánica prolongada ${ }^{1}$. Sin embargo, también afecta la posibilidad del paciente para comunicarse efectivamente a través del habla, ya que el flujo de aire no se realiza a través de las cuerdas vocales ${ }^{2,3}$.

Así, fue creada la válvula de fonación (VF), dispositivo que se instala sobre la cánula de traqueostomía (CTQT) que permite flujo unidireccional durante la inspiración, y que se cierra o se mantiene cerrada durante la fase espiratoria causando la redistribución del gas exhalado hacia la laringe, boca y cavidad nasal ${ }^{3-5}$. La generación de presión positiva en la vía aérea y posterior flujo ascendente hacia el exterior, no sólo permite la fonación, sino que también tendría efectos positivos sobre el mecanismo de deglución, disminuyendo el riesgo de aspiración ${ }^{2,5-7}$.

La broncoscopía es el mejor método para evaluar la permeabilidad de la vía aérea ${ }^{3,8}$, sin embargo, se trata de un procedimiento invasivo, realizado por un especialista que requiere sedación profunda o anestesia general, con todos los riesgos que ello implica. Como alternativa existen evaluaciones no invasivas de la permeabilidad de la vía aérea como la auscultación antes y durante la oclusión de la CTQT, auscultación mientras se usa la VF, evidenciar el aire exhalado a través de la boca y nariz, y su medición objetiva a través de la espirometría. Sin embargo, muchos niños son muy pequeños para seguir instrucciones, de manera que algunos autores han planteado medir la presión a través de la CTQT mientras el paciente usa la VF, siendo una prueba práctica, simple y segura ${ }^{2-4}$.

En estudios similares, se utiliza el término de presión al final de espiración (PFE), que representa la presión al final de la espiración fisiológica, que puede ser cero. Nuestro objetivo es conocer la presión que se mantiene durante la espiración tranquila del paciente, por lo que preferimos utilizar el término de "presión espiratoria mantenida" (PEMant).

De esta forma, la medición de PEMant pudiera ser un indicador clínico de la permeabilidad de la vía aérea (VA).

\section{Material y Método}

Se estudiaron pacientes traqueostomizados en el Hospital Josefina Martínez, durante doce meses. La evaluaciones fueron realizadas a solicitud del médico tratante.

\section{Criterios de inclusión}

Pacientes con traqueostomía por un período mayor o igual a 30 días, sin necesidad de ventilación mecánica o con ventilación mecánica parcial (sólo nocturna o que toleren períodos de desconexión) y clínicamente estable por más de 2 semanas.

\section{Criterios de exclusión}

Pacientes con intercurrencia respiratoria (dificultad respiratoria, requerimientos adicionales de $\mathrm{O}_{2}$, aumento de los parámetros de VM, fiebre).

\section{Procedimiento de evaluación}

- Evaluación inicial: Registro de variables clínicas $\left(\mathrm{SaO}_{2}\right.$, frecuencia cardíaca (FC) y respiratoria (FR), uso de musculatura accesoria y signos de resistencia de la VA) (Tabla 1) previo al uso de VF.

- Evaluación con VF: Se instaló la VF (SSVO Phonate $^{\mathrm{TM}}$ Speaking Valve with Oxygen Port, Shiley, Tyco healthcare Group LP), conectada a un manómetro aneroide (Mod. EN 837-3, Tecsis, MULTEC, 0-160 $\mathrm{cmH}_{2} \mathrm{O}$ ) a través de una silicona de $20 \mathrm{~cm}$ desde su puerto de $\mathrm{O}_{2}$ (Figura 1). Luego, se registró la PEMant durante 60 segundos a los minutos 1, 5, 10 y 15, definiendo el valor de PEMant como la moda durante cada uno de esos 60 s. Además, se registraron las variables clínicas en cada tiempo de evaluación. Se suspendió la evaluación a los 15 minutos o antes si presentó tos persistente, incomodidad, llanto, excesiva cantidad de secreciones, registro de PEMAnt mayor a $40 \mathrm{cmH}_{2} \mathrm{O}$ y/o uno de los siguientes: $\mathrm{SaO}_{2}$ $<93 \%$, FC sobre el 20\% de su basal, FR elevada para la edad, uso de musculatura accesoria o aumento de la resistencia en la VA.

Tabla 1. Variables clínicas de registro

\begin{tabular}{|llc|}
\hline $\begin{array}{l}\text { Uso de musculatura } \\
\text { accesoria }\end{array}$ & $\begin{array}{l}\text { Resistencia de } \\
\text { la vía aérea }\end{array}$ & Puntos \\
$\begin{array}{l}\text { Retracción } \\
\text { intercostal }\end{array}$ & $\begin{array}{l}\text { Espiración } \\
\text { prolongada }\end{array}$ & 1 \\
$\begin{array}{l}\text { Uso musculatura } \\
\text { supraclavicular }\end{array}$ & $\begin{array}{l}\text { Sibilancias } \\
\text { espiratorias }\end{array}$ & 2 \\
$\begin{array}{l}\text { Uso de musculatura } \\
\text { abdominal }\end{array}$ & $\begin{array}{l}\text { Sibilancias en } \\
\text { ambas fases }\end{array}$ & 3 \\
\hline
\end{tabular}




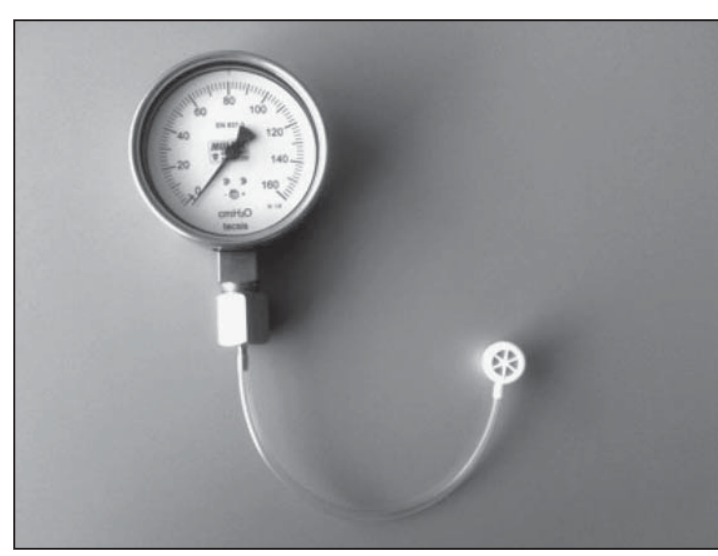

Figura 1. Sistema de medición de PEMant. Manómetro aneroide conectado a válvula de fonación.

\section{Método} dinal.

Estudio de tipo prospectivo de corte longitu-

Luego de la evaluación, los pacientes fueron divididos en 2 grupos de acuerdo a la tolerancia al uso de la VF: los que completaron la prueba se denominó Grupo de tolerancia (GT), y los que presentaron alguno de los signos de dificultad respiratoria en algún momento de la evaluación, se denominó Grupo de no tolerancia (GNT)

Para el análisis de resultados se utilizó el programa estadístico SPSS 17.0. El análisis descriptivo es expresado a través de medidas porcentuales, mediana y rango, según corresponda. Para comparaciones intergrupo se utilizó el test de Mann-Whitney y el de Wilcoxon para muestras pareadas. Fue considerado como significativo un valor de $\mathrm{p}<0,05$.

\section{Resultados}

Se evaluó a un total de 23 niños, trece pacientes de sexo masculino.

Por grupos, se observó un total de 10 pacientes en GT y 13 pacientes en GNT. Al analizar las edades, la mediana global fue de 22 meses (rango 6-159 meses), al comparar grupos se evidenció una diferencia significativa entre éstos, siendo menores los pacientes del GNT. En ambos grupos se presentan las indicaciones más habituales de traqueostomía: obstrucción de vía aérea superior crítica (estenosis subglótica, parálisis de cuerdas vocales) y necesidad de ventilación mecánica prolongada (enfermedad neuromuscular, bronquiolitis obliterante, displasia broncopulmonar, hernia diafragmática, traqueobroncomalacia). Junto con los datos demográficos, se muestran las enfermedades de base en la Tabla 2.
En cuanto a valores de PEMant, se observó que en el GT los valores fueron menores a 10 $\mathrm{cmH}_{2} \mathrm{O}$ y toleraron de manera adecuada la VF, en cambio, en GNT los valores de PMant se registraron por sobre los $20 \mathrm{cmH}_{2} \mathrm{O}$ y no toleraron el uso de VF, encontrando diferencias significativas entre ambos grupos, para valores de PMant inicial y final (Tabla 3).

No se encontró diferencias significativas en los valores de $\mathrm{SaO}_{2}$ inicial o final en la comparación entre los grupos, a pesar de la tendencia a presentar $\mathrm{SaO}_{2}$ más bajas en la evaluación final en el GNT. Tampoco se encontró diferencias en valores de FC inicial o final en ambas comparaciones, a pesar de la tendencia en GNT a presentar en la evaluación final FC más elevadas. En la evaluación inicial, la FR es más alta en GNT en forma significativa $(p=0,012)$, pero no asi en la evaluación final. Respecto del uso de musculatura accesoria (UMA), no se encontró diferencia significativa en la evaluación inicial, sin embargo en la evaluación final, el GNT mostró un índice significativamente mayor que el del GT $(p=0,03)$. No hubo diferencia en cuanto a la aparición de sibilancias como marcador de resistencia de vía aérea, al comparar los grupos (Tabla 4).

A analizar el GNT, pudimos observar que sólo seis de trece pacientes toleraron hasta el minuto 1 , dos pacientes hasta el minuto 5 , tres

Tabla 2. Características clínicas

\begin{tabular}{|lccc|}
\hline & GT & GNT & Total \\
Edad (meses) & 10 & 13 & 23 \\
& $\begin{array}{c}37,5^{*} \\
(14-159)\end{array}$ & $\begin{array}{c}20^{*} \\
(6-75)\end{array}$ & $\begin{array}{c}22 \\
(6-159)\end{array}$ \\
Sexo (masculino) & 7 & 6 & 13 \\
Indicacion de TQT & & & \\
OVAS & 4 & 5 & 9 \\
$\quad$ ESG & 4 & 3 & 7 \\
$\quad$ PCV & 0 & 2 & 2 \\
Necesidad de VMP & 6 & 8 & 14 \\
$\quad$ ENM & 4 & 0 & 4 \\
BO & 1 & 0 & 1 \\
DBP & 0 & 1 & 1 \\
HDC & 0 & 1 & 1 \\
TBM & 1 & 6 & 7 \\
\hline
\end{tabular}

GT: Grupo que tolera, GNT: Grupo que no tolera, TQT: traqueostomía, OVAS: obstrucción de vía aérea, ESG: estenosis subglótica, PCV: parálisis de cuerdas vocales, VMP: ventilación mecánica prolongada, ENM: enfermedad neuromuscular, BO: bronquiolitis obliterante, DBP: displasia broncopulmonar, HDC: hernia diafragmatica congénita, TBM: traqueobroncomalacia. ${ }^{*} \mathrm{p}<0,03$. 
Tabla 3. Valores (moda) de presión mantenida de vía aérea $\left(\mathrm{cmH}_{2} \mathrm{O}\right)$

\begin{tabular}{|ccccc|}
\hline & GT & GNT & Total & P \\
Minuto 1 & & & & \\
n & 10 & 13 & 23 & \\
mediana & $5(2-18)$ & $30(19-80)$ & & $<0,001$ \\
Minuto 5 & & & & \\
n & 10 & 7 & 17 & \\
mediana & $4(2-16)$ & $26(12-30)$ & & $<0,001$ \\
Minuto 10 & & & & \\
n & 10 & 5 & 15 & \\
mediana & $7(2-10)$ & $22(19-40)$ & & $<0,001$ \\
Minuto 15 & & & & \\
n & 10 & 2 & 12 & \\
mediana & $6(2-10)$ & $31(22-40)$ & & $<0,001$ \\
\hline
\end{tabular}

pacientes hasta el minuto 10, y sólo dos toleraron con esfuerzo hasta el minuto 15. Las presiones registradas en estos pacientes que terminaron con anterioridad la prueba son mayores que las registradas en los que lograron finalizarla.

\section{Discusión}

$\mathrm{Al}$ revisar la literatura, se evidencia que existe información variada de valores de presión de vía aérea para usar como referencia. Hess et al y Brigger et al, se refieren a resistencia espiratoria excesiva con valores sobre $5 \mathrm{cmH}_{2} \mathrm{O}$ durante la exhalación pasiva en adultos ${ }^{2,5}$, Brigger et al y Gerau et al, establecen que una de las indicaciones para el uso de VF es la medición de presiones traqueales menores a $10 \mathrm{cmH}_{2} \mathrm{O}^{2,9}$.

Utrarachkil et al, evaluaron a 22 niños traqueostomizados durante 5 minutos, encontrando que éstos toleraban el uso de VF con valores de presión al final de la espiración (PFE) de 2-6 $\mathrm{cmH}_{2} \mathrm{O}$, mientras que aquellos con PFE mayores a $10 \mathrm{cmH}_{2} \mathrm{O}$ demostraron tener dificultad respiratoria ${ }^{3}$. Holmgren et al evaluó a los pacientes a los minutos 1,5 y 10 posterior a la instalación de la VF, este único estudio nacional, además establece que valores de $\mathrm{PFE}$ menores a $12 \mathrm{cmH}_{2} \mathrm{O}$ sugieren buena tolerancia, no siendo necesaria una evaluación endoscópica de rutina previo a su uso ${ }^{4}$.

Luego de analizar los valores obtenidos, podemos inferir que valores de PEMant menores a $10 \mathrm{cmH}_{2} \mathrm{O}$ con VF sugieren buena tolerancia a ésta. A su vez, PEMant mayor a $20 \mathrm{cmH}_{2} \mathrm{O}$ sugieren que el paciente no tolerara la VF. Así,
Tabla 4. Variables clinicas registradas

\begin{tabular}{|lccc|}
\hline & GT & GNT & P \\
$\mathrm{SaO}_{2}$ inicial & $97(93-99)$ & $97(94-100)$ & NS \\
$\mathrm{SaO}_{2}$ final & $96,5(95-98)$ & $93(78-100)$ & NS \\
FC inicial & $114(72-140)$ & $117(94-140)$ & NS \\
FC final & $115(77-148)$ & $125(104-147)$ & NS \\
FR inicial & $25(20-47)$ & $37(24-72)$ & NS \\
FR final & $28(17-49)$ & $43(17-60)$ & NS \\
UMA inicial & $0(0-1)$ & $0(0-1)$ & NS \\
UMA final & $0(0-1)$ & $2(0-3)$ & 0,03 \\
RVA inicial & $0(0-0)$ & $0(0-2)$ & 0,012 \\
RVA final & $0(0-1)$ & $0,5(0-3)$ & NS \\
\hline
\end{tabular}

GT: Grupo que tolera, GNT: grupo que no tolera, $\mathrm{SaO}_{2}$ : saturacion arterial de oxígeno, FC: frecuencia cardiaca, FR: frecuenca respiratoria, UMA: uso de musculatura accesoria, RVA: resistencia de vía aérea. Todos los valores expresados en mediana y (rango).

con esta prueba de presiones que puede predecir la tolerancia a la válvula, se puede diferir y eventualmente evitar la evaluación endoscópica de la vía aérea.

El rango de PEMant de 10 a $20 \mathrm{cmH}_{2} \mathrm{O}$ no cuenta con datos que respalden su utilidad como predictor de tolerancia. Es probable que el aumento del tamaño muestral permita reducir este rango de incertidumbre.

Las variables de PEMant, FR y UMA, fueron mayores en el GNT, sin embargo, sólo el UMA fue significativamente mayor en la evaluación final. En otros estudios se utilizaron válvulas Passy Muir ${ }^{\mathrm{TM} 3,4}$ las cuales utilizan el mecanismo de cierre del diafragma denominado "positive closure" que a diferencia de la válvula SSVOTM Shiley, no fuga aire en la fase espiratoria, lo que explicaría nuestro hallazgo del aumento del esfuerzo muscular evidenciado por UMA, especificamente uso de musculatura supraclavicar. Sin embargo, en dichos estudios previos con VF Passy MuirTM ${ }^{3,4}$ no describen el comportamiento de la musculatura respiratoria, siendo necesario comparar ambos tipos de VF.

De esta forma, el esfuerzo muscular es lo que condicionaría en mayor medida la tolerancia o no al uso de VF, siendo este elemento de medición el más relevante a la hora de determinar conductas. Además, cabe destacar la riqueza de una evaluación clínica más completa, que incluyó FC, $\mathrm{SaO}_{2}$, FR, UMA y RVA, que representa mejor la condición global del paciente.

Probablemente pacientes que no toleran la VF por falta de permeabilidad en su vía aérea lo 
dejarán de manifiesto de manera precoz, por lo que es lógico esperar que exista esta diferencia en la PEMant inicial y, asociado a ello, un aumento de la FR.

A diferencia de otros estudios, el nuestro realizó medición de PEMant hasta los 15 minutos, permitiendo una ventana de observación clínica mayor, pudiendo otorgar mayor sensibilidad a esta prueba.

Pese a que ambos grupos presentaban diagnósticos de obstrucción de vía aérea similar, los valores de PEMant y la tolerancia fue dispar. Consideramos que ésto puede explicarse por la mejoría espontánea en la obstrucción de la vía aérea, la que no ha sido evidenciada por estudio endoscópico.

Finalmente, proyectamos completar este estudio correlacionando los hallazgos con una evaluación endoscópica de la vía aérea, para establecer definitivamente la utilidad de la PEMant como indicador clínico de la permeabilidad de la vía aérea para uso de VF.

\section{Referencias bibliográficas}

1.- DURBIN C G Jr. Indications for and timing of tracheostomy. Respir. Care 2005; 50: 483-7.

2.- HESS D. Facilitating Speech in the Patient with a Tra- cheostomy. Respir Care 2005; 50: 519-25.

3.- UTRARACHKIJ J, PONGSASNONGKUL J, PREUTTHIPAN A, CHANTAROJANASRI T. Measurement of End-Expiratory Pressure as an Indicator of Airway Patency above Tracheostomy in Children. J Med Assoc Thai 2005; 88: 928-33.

4.- HOLMGREN L, BROCKMANN P, BERTRAND P. Factor Predictor de tolerancia a válvula de fonación (VF) en pacientes traqueostomizados. Resúmenes presentados en el V Curso Internacional y II Congreso de Neumología Pediátrica, Santiago de Chile. Rev Neumología Pediátrica 2007; 2: 157.

5.- BRIGGER M, HARTNICK C. Drilling speaking valves: A modification to improve vocalization in tracheostomy dependent children. Laryngoscope, 2009; 119: 176-9.

6.- Kent L. Tracheostomy Decannulation. Respiratory Care 2005; 50: 538-41.

7.- PAZ F, ZAMORANO A, PAIVA R, HERNÁNDEZ Y, MÖDINGER P, MOSCOSO G. Cuidados de niños con traqueotomía. Rev Neumología Pediátrica 2008; 3: 6470.

8.- NUSSBAUM E. Usefulness of Miniature Flexible Fiberoptic Bronchoscopy in children. Chest 1994; 106; 1438-42.

9.- GEREAU S, NAVARRO G, CLUTERIO B, MULLAND E, BASSILA M, RUBEN R. Selection of pediatrics patients for use of the Passy-Muir valve for speech production. International J Ped Otorhinolaryngology 1996; 35: 11-7.
Correspondencia a:

Klgo. Gregory Villarroel S.

Hospital Josefina Martínez

Dirección: Av. Camilo Henríquez \# 3691, Puente Alto.

Santiago, Chile.

E-mail: gregoryvs@gmail.com 